The submitsed manuscript has been

authured by $n$ contractur if the U.S. Guvernment under contract $D E$. ACO5-84(1)R21400. Accordingly, the U.S. Guvernment retains n nonexclusive. royalty-free license to publish or reproduce the published form of this cuntribution. ur allow othere so do so. fur U.S. Guvernment purpusen."

\title{
THREE-DIMENSIONAL CALCULATIONS OF FIELDS AND LOADING FOR LOOP AND FOLDED WAVEGUIDE ICRF ANTENNAS*
}

\author{
M. D. Carter, F. W. Baity, D. B. Batchelor, D. J. Hoffman, \\ E. F. Jaeger, D. W. Swain and G. R. Haste \\ Oak Ridge Natioral Laboratory, Oak Ridge, Tennessee 37831

\section{INTRODUCTION}

The ANT and ORION codes have been combined and used to study the loading of various antenna geometries with simple three-dimensional (3-D) effects. Both codes use a slab model with periodic Fourier analysis for modeling the toroidal and poloidal directions. The ANT code is used to prescribe current sets in a vacuum region where the field solutions are obtained analytically for each Fourier mode and matching conditions are used at poloidal/toroidal current sheet locations. Multiple current sheets are permitted and various feeder options are available to model the radial antenna currents. Current elements may be oriented at arbitrary angles to the static inagnetic field and may be independently phased in time. The fields at the plasma surface are prescribed by an impedance matrix for each Fourier mode. The ORION code solves for the fields in the plasma region using finite difference techniques, a plasma dispersion relation that retains the lowest-order finite gyroradius effects (although only cold plasma is considered here), and all three electric field components [see E. F. Jaeger, D. B. Batchelor, and H. Weitzner, Nucl. Fusion 28, 53 (1988)]. Results are presented for a folded waveguide mock-up and for a loop antenna design under identical plasma conditions.

\section{ANT CODE DESCRIPTION}

The current element geometry is represented in the ANT code using patch functions. These patch functions have an analytic value of one within a finite rectangular region in the $y$ (poloidal) and $z$ (toroidal) directions. Outside this region, the analytic value is zero. The Fourier coefficients for these elements are calculated analytically so as to obtain the exact value for the retained coefficients. Currents are allowed tc flow along one axis of these patch functions, and this axis may be rotated by an arbitrary angle in a constant $x$ (radial) plane. Current feeders are implemented to carry currents in the $x$ direction by using Fourier representations of delta functions at each end of a current element. Presently, these feeders may be absent, have constant current in the radial direction, or have a half-wavelength standing wave pattern between the element and the conducting back-plane.

Maxwell's equations for a vacuum region are solved analytically with conducting boundary conditions at the back-wall along with continuous electric

* Research sponsored by the Office of Fusion Energy. U.S. Department of Energy, under contract DE-AC05-84OR21400 with Martin Marietta Energy Systems, Inc. 
fields and the appropriate jump conditions for derivatives across each current element. Faraday's law and an impedance matrix generated by the ORION code for each Fourier mode are used at the plasma interface.

\section{FOLDED WAVEGUIDE USING "EQUIVALENT CURRENT SETS"}

For the folded waveguide studies, the plasma is held invariant while the amplitude and direction of the current elements are manually adjusted until the boundary conditions in the waveguide are roughly satisfied for the magnetic field and for the poloidal and toroidal components of the electric field. The current elements produce a current sheet at the polarizing plate location and exposed sides of the waveguide. The feeders for these elements are positioned to coincide with the vanes of the waveguide. The power is generated primarily by the feeder elements $\left(\int E_{x} J_{x}^{*} d V\right)$ in reasonably converged cases using a half-wavelength current distribution for the feeders. This modeling is shown schematically in Fig. 1.

The estimate of the electric field at the quarter-wavelength point of the waveguide is crucial in making contact with experimental measurements on folded waveguides, and requires the use of the appropriate feeder length in the model. A rough formula based on a resonant cavity is $(2 \mathrm{f} / \mathrm{c})^{2}=W^{-2}+L^{-2}$, where $W$ is roughly the waveguide width times the number of folds, $L$ is the length of the waveguide, $f$ is the resonant frequency in cycles per second, and $c$ is the speed of light. In the actual device, a polarizing plate at one end of the structure covers alternating regions between the waveguide vanes and allows currents to close in a desirable direction for launching waves into the plasma.

To model the folded waveguide, each polarizing plate of the waveguide was represented by 15 current elements, with 2 additional elements placed on each exposed section of the side-walls of the waveguide. The plate elements spanned $7 \mathrm{~cm}$ in the poloidal direction and covered $32 \mathrm{~cm}$ in the toroidal direction. Five sets of these current elements were spaced poloidally with $7 \mathrm{~cm}$ separation, and elements were eliminated outside a poloidal range of $70 \mathrm{~cm}$. Thus, the model simulates a $32 \times 70 \mathrm{~cm}$ waveguide having 10 folds, 5 of which are alternately covered by polarizing plates. The initial guess for the currents on these elements followed a cosine distribution as if the waveguide were not folded.

Power coupling was estimated using a plasma with a periodic toroidal length of $2 \pi(2.15) \mathrm{m}$, a periodic poloidal length of $2 \pi(0.75) \mathrm{m}$, and a magnetic field of $8.5 \mathrm{~T}$ on axis (6.3 $\mathrm{T}$ near the antenna). The deuterium plasma density was $2.5 \times 10^{20}$ $\mathrm{m}^{-3}$ on axis with a square root parabolic profile out to the plasma separatrix, where the density was $2.5 \times 10^{19} \mathrm{~m}^{-3}$. The scrape-off plasma was $4 \mathrm{~cm}$ wide with a $2 \mathrm{~cm}$ e-folding length to produce a density of $3.4 \times 10^{18} \mathrm{~m}^{-3}$ at the plasma edge. The $\mathrm{rf}$ frequency was taken to be $86.4 \mathrm{MHz}$. The waves were completely absorbed after several fast-wave wavelengths by a diffuse artificial absorber in the ORION code, and 161 poloidal modes and 201 toroidal modes (ranging from -80 to 80 and -100 to 100 , respectively) were used. The radial length of the waveguide was $2.1 \mathrm{~m}$ at $86.4 \mathrm{MHz}$. Note that this model uses a higher frequency and a length that is about $20 \%$ shorter than the experimental waveguide mock-up that was tested at roughly $78 \mathrm{MHz}$ in the RF Test Facility (RFTF) at ORNL. ${ }^{1}$ The model results required the amplification and toroidal redistribution of the 
poloidal component of the current elements when the plasma edge was very near the polarizing plate.

For the waveguide current set model used here, $\int E_{y} J_{y}^{*} d V^{-}$contributed less than $10 \%$ of the total power produced, with roughly $90 \%$ of the power generated by the feeders. This low value of $\int E_{y} J_{y}^{*} d V$, in conjunction with visual inspection in real space using the Ximage software by NCSA, indicated that the tangential electric field on the polarizing plate surface is very nearly zero for the current set. The loading calculation was performed relative to the location of peak electric field in the waveguide (a quarter-wavelength back from the polarizing plate). Ximage was used to estimate the average electric field between the vane tip and the nearby vanes. Note that peaking near the vane tips was limited in the calculation because of the 2 to $3 \mathrm{~cm}$ resolution using a finite number of Fourier modes; however, the limited resolution is expected to produce errors in the average electric field on the order of 10 to $20 \%$.

The power launched by the waveguide into the plasma was estimated in three different ways to verify consistency of the solutions. The first was through the volume integration of $E \cdot J^{*}$ using analytic values in the $x$ (radial) direction and summing over all Fourier harmonics. The second method was to calculate the integral of the Poynting flux over the entire plasma surface using Parseval's theorem in Fourier space. These two methods of power calculation agree with one another to within the expectations of digital rounding errors, suggesting that the field solutions have been accurately programmed. The third estimate involved integration of the Poynting flux in real space over a region extending from $-72 \mathrm{~cm}$ to $72 \mathrm{~cm}$ in both the poloidal and toroidal directions and centered on the center of the waveguide structure using a $2 \mathrm{~cm}$ real-space grid. This estimate indicates the power launched through a restricted region of real space and also provides an independent estimate to verify the accuracy of the inverse Fourier interpolation of the fields into real space. The real-space power reasonably matched the calculations in Fourier space. The power coupled into the plasma described above by this current set was $0.014 \mathrm{~W} /(\mathrm{V} / \mathrm{cm})^{2} \pm 30 \%$ using the maximum average vaneto-vane electric field (at the quarter-wavelength point). The field intensities at the plasma surface for this case ( $1 \mathrm{~mm}$ in front of the polarizing plate) are shown in Fig. 2. Note that the grey scale used for this figure does not properly distinguish the sign of the field components, but does indicate the shape of the patterns.

The maximum electric field in the waveguide (averaged over the distance between vanes) that was experimentally tested on the RFTF was estimated to be roughly 21 to $23 \mathrm{kV} / \mathrm{cm}$ at $78 \mathrm{MHz} .{ }^{1}$ Using this experimentally realized value of the average electric field at the center of the waveguide indicates that roughly $7 \mathrm{MW}$ of power could be launched into a plasma having the previously described profile model. This represents a power density of roughly $31 \mathrm{MW} / \mathrm{m}^{2}( \pm 30 \%)$ for this waveguide model at $86.4 \mathrm{MHz}$. Of course, a large source of error in this estimate also comes from the lack of knowledge concerning the plasma profile in a real experiment, and some comparison with loop antenna designs is desirable.

\section{PRELIMINARY LOOP ANTENNA CALCULATIONS}

It is difficult to make a direct comparison of the waveguide and loop antenna structures because of the different considerations in their design; however, modeling of a loop antenna design for the same plasma parameters as were used 
for the folded waveguide is possible. To model the currents in a proposed wallmounted antenna design for BPX, a set of eight current elements was arranged with four elements each at two toroidal locations and with poloidal stacking of the elements to produce a nearly constant poloidal current with roughly $1 \mathrm{~m}$ of poloidal extent. Each element was $14.8 \mathrm{~cm}$ wide and $23 \mathrm{~cm}$ long with a uniform current distribution. The two sets were separated toroidally by $30.5 \mathrm{~cm}$ and driven $180^{\circ}$ out of phase at $86.4 \mathrm{MHz}$. The actual design proposes a septum between the elements; however, the image current carried in the septum is zero in a $180^{\circ}$ phasing scenario. The straps were placed $3.4 \mathrm{~cm}$ from the front of the Faraday screen and $26.6 \mathrm{~cm}$ from the grounded back-wall, and the feeders were modeled with constant iadial currents. Side-wall return currents were modeled by placing additional 5 - $\mathrm{cm}$-wide straps toroidally separated by $62 \mathrm{~cm}$ in the same plane as the main driving elements. Each of these side-wall elements carried one-half the current of the main driving elements. Note that the radiation from the feeders in this model is negligible compared to that from the straps. The Faraday screen was not explicitly modeled, but was taken to be located at the plasma edge in the above plasma model. This simple current set radiated $12.7 \mathrm{~W}$ of power into the plasma for $1 \mathrm{~A}$ of current in each strap (peak value in time). The field intensities for the loop antenna are shown in Fig. 3. Again, note that the grey scale used for this figure does not properly distinguish the sign of the field components.

The return currents in the top of the structure and finite phase velocity effects can be estimated using length corrections for the design. ${ }^{2}$ An approximate correction based on these considerations indicates a reauction in power by a factor of roughly 0.8 for the actual proposed design. Using an estimate of $1000 \mathrm{~A}$ for the actual peak current, this structure would launch on the order of $10 \mathrm{MW}$, but the area of this system is roughly 3.5 times that of the folded waveguide structure discussed above, yielding a power density of roughly $12 \mathrm{MW} / \mathrm{m}^{2}$. This value is a factor of 2.5 lower than that obtained for the folded waveguide current set.

\section{CONCEUSIONS}

The combined ANT/ORION code at ORNL is evolving into a useful tool to study ICRF launching structures. The present modeling efforts have provided some indications for improvement in folded waveguide designs and show that present designs may have significant advantages in terms of power density over loop antenna designs. The modeling with plasma in conjunction with experimental testing indicates that a waveguide structure should have on the order of two to three times the power handling capabilities of loop antenna designs.

\section{REFERENCES}

1. F. W. Baity, T. S. Bigelow, M. D. Carter, W. L. Gardner, R. H. Goulding, G. R. Haste, D. J. Hoffman, and D. O. Sparks, paper B 15 presented at the Ninth Topical Conference on Radio Frequency Power in Plasmas, August 1921, 1991, Charleston, SC.

2. P. M. Ryan, in Proceedings of the 18th European Conference on Controlled Fusion and Plasma Physics, Berlin, June 3-7, 1991, edited by P. Bachmann and D. C. Robinson (European Physical Society, Petit-Lancy, Switzerland, 1991), Vol. 15C, Part III, p. 309. 


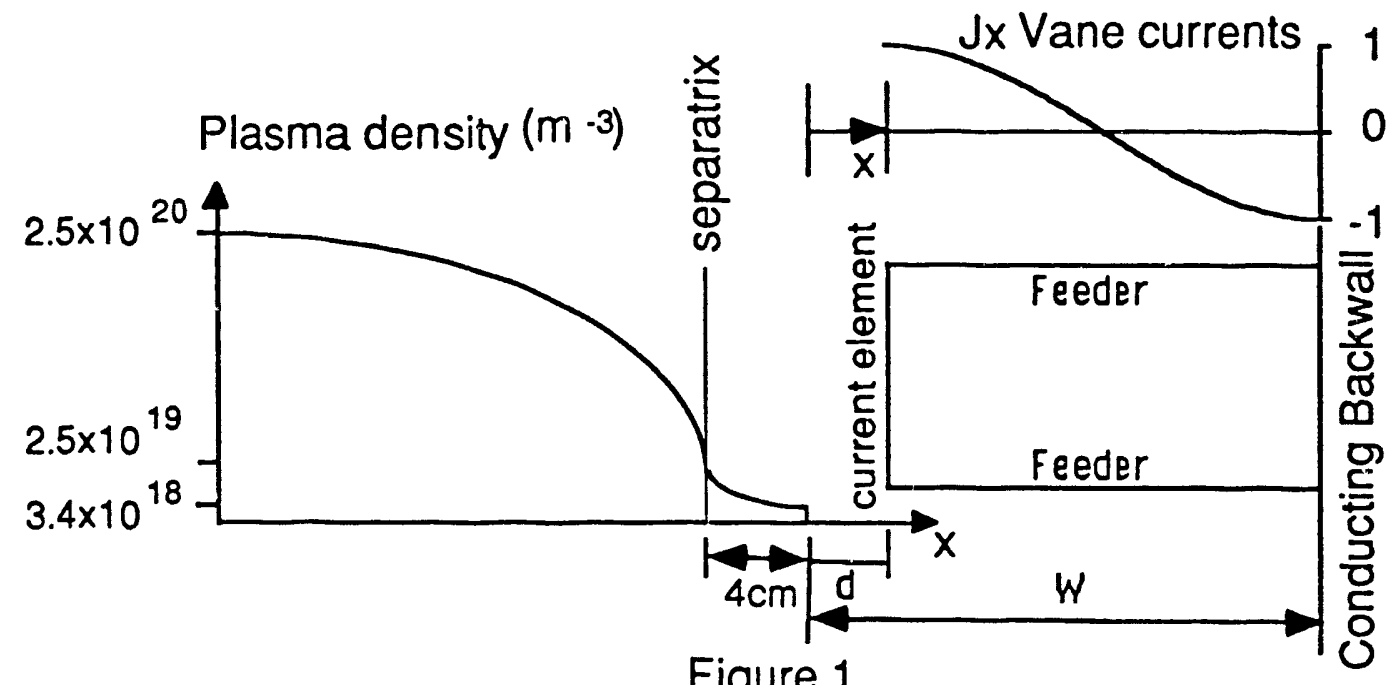

Figure 1

Folded Waveguide at Plasma Surface
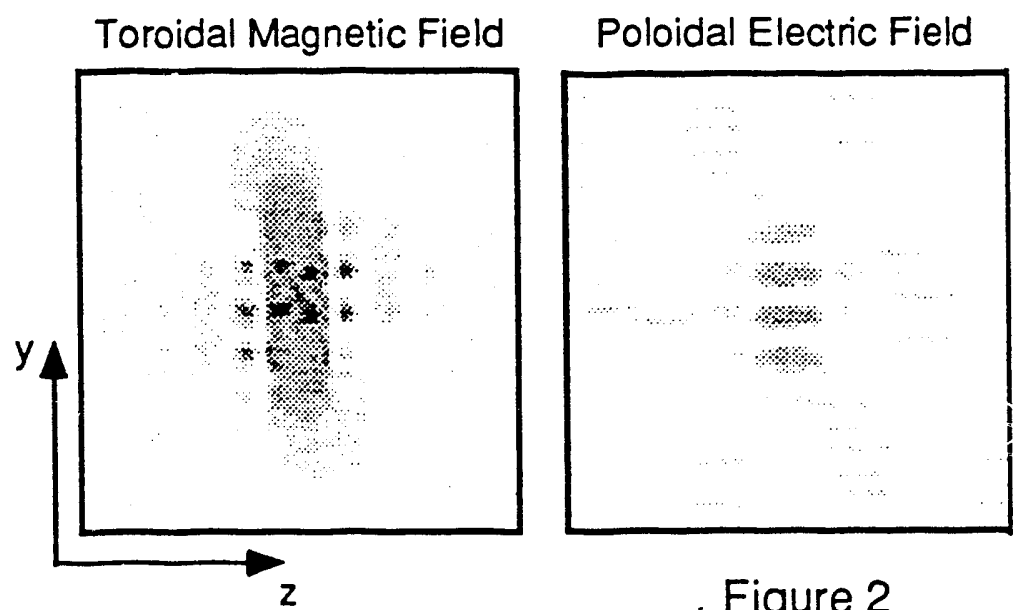

Poynting Flux

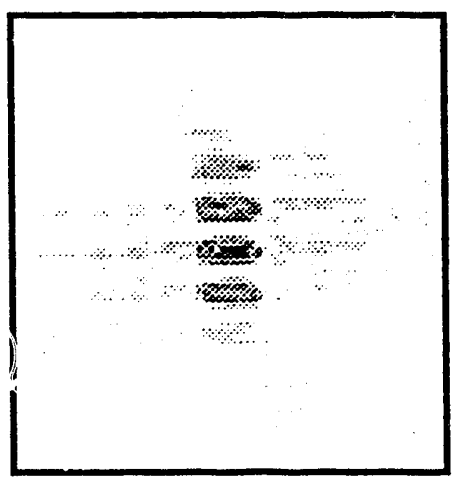

Figure 2

Loop Design at Plasma Surface
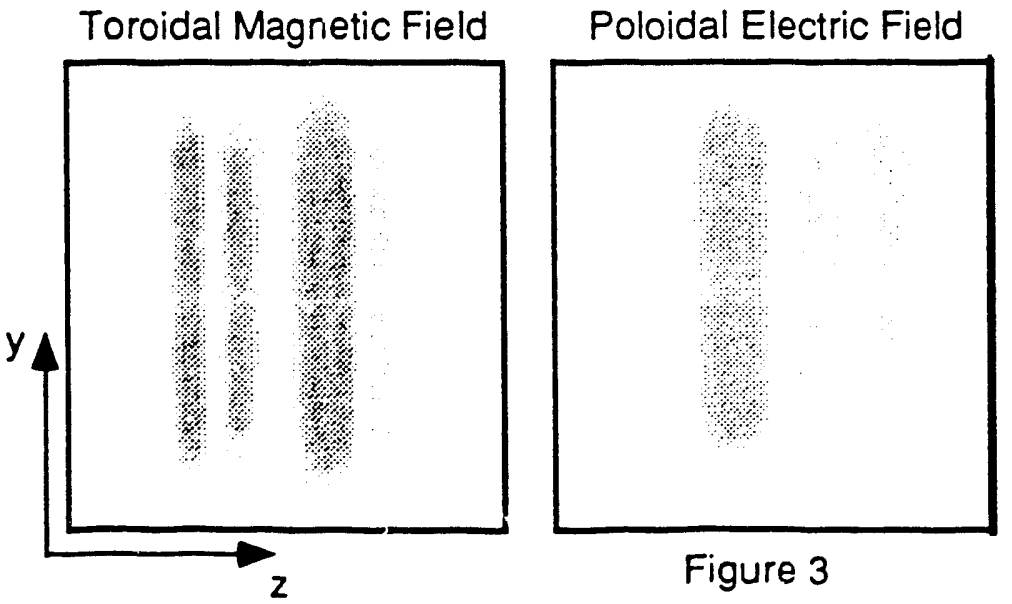

Poynting Flux

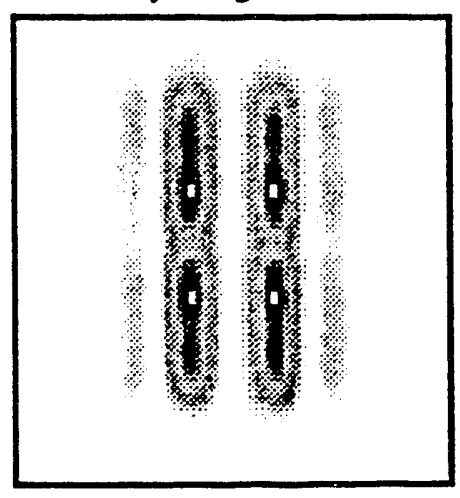

Figure 3 


\section{DISCLAIMER}

This report was prepared as an account of work sponsored by an agency of the United States Governinent. Neither the United States Government nor any agency thereof, nor any of their employees, makes any warranty, express or implied, or assumes any legal liability or responsibility for the accuracy, iompleteness, or usefulness of any information, apparatus, product, or process dis ilosed, or represents that its use would not infringe privately owned rights. Refererce herein to any specific commercial product, process, or service by trade name trademark, manufacturer, or otherwise does not necessarily constitute or imply iss endorsement, recommendation, or favoring by the United States Governmen' or any agency thereof. The views and opinions of authors expressed herein do not necessarily state or reflect those of the United States Government or any agency thereof. 

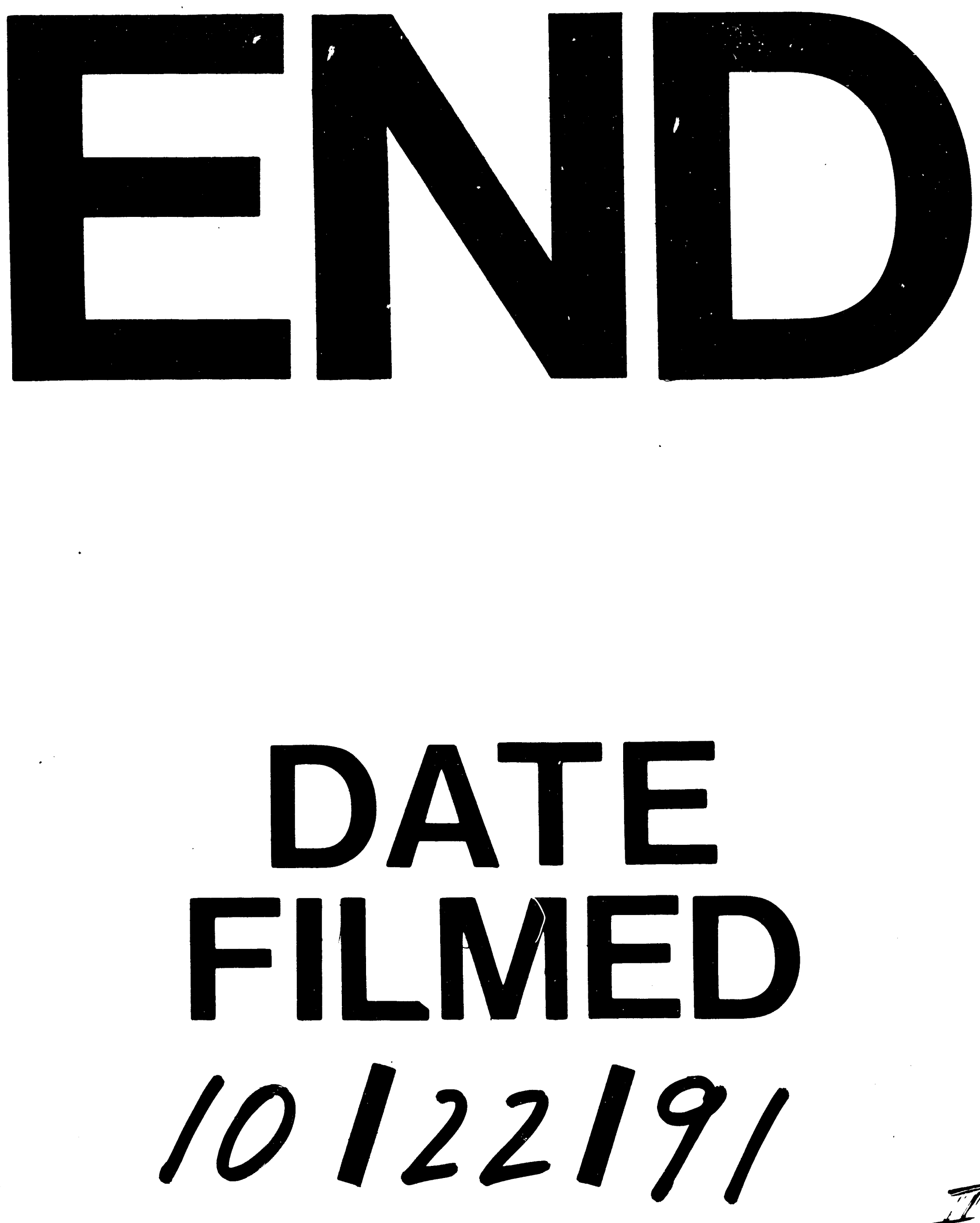
